\title{
The evolutionary dynamics of cancer prevention
}

\author{
Robert A. Gatenby, MD, \\ Departments of Radiology and Integrated Mathematical Oncology at the Moffitt Cancer Center \\ Robert J. Gillies, PhD, and \\ Departments of Radiology and Integrated Mathematical Oncology at the Moffitt Cancer Center \\ Joel S. Brown, PhD \\ Department of Ecology and Evolutionary Biology at the University of Illinois
}

\section{Commentary}

The human body plays with evolutionary fire.

Optimal function requires large specialized cellular populations continuously replenished by rapidly proliferating stem cells. But, every cell division risks initiation of evolutionary events leading to uncontrollable proliferation and a fatal malignancy. Cancer is, in other words, the price of multicellularity.

Carcinogenesis is well recognized as "somatic evolution", but it is actually two competing evolutionary processes: (1) potential cancer-forming cells evolve adaptations to overcome the tumor suppression mechanisms of the whole organism; and (2) the organisms themselves evolving strategies to reduce the probability of death from cancer prior to and during reproductive maturity.

At first glance, this seems an uneven contest. Whereas organisms can contribute successful cancer-prevention strategies to subsequent generations; each cancer must "reinvent the wheel", as its evolutionary products die with the host. However, these Darwinian processes are deeply interconnected as each cancer cell has at its disposal the full complement, complexity and adaptability of the host genome. For example, simply upregulating or downregulating existing genes can explore cellular phenotypes and functions that are ordinarily beyond the reaction norm of differentiated cells. Thus, the genomic information and plasticity of ever-more complex multicellular organisms also increases the range of phenotypes and adaptive strategies available to evolving cancers that originate from that organism's cells.

That cancer is largely a disease of old age indicates humans have evolved mechanisms to suppress carcinogenesis prior to reproduction. So, in this evolutionary arms race between multicellular organisms and their cancers, what strategies are evident?

There are two requirements for a cell to begin somatic evolution to a cancer. First, it must have a self-defined fitness function wherein cells proliferate based on their own evolved phenotypic properties unlike normal cells whose proliferation is entirely dependent on local tissue growth controls. Second, it requires an environment within which it can proliferate and attain a sufficient population size for the accumulation of new mutations.

Corresponding Author: Robert A. Gatenby, MD, Departments of Radiology and Integrative Mathematical Oncology, 12902 Magnolia Dr, Tampa, FL 33612, Phone (813) 745-2843, Robert.Gatenby@ Moffitt.org. 
Clearly epithelial surfaces present great cancer risks. The colon surface, for example, consist of perhaps $10^{11}$ continuously renewing cells adjacent to a lumen that provides vast potential space for population expansion and subjected to a wide range of environmental perturbations. Given this enormous evolutionary opportunity, the prevalence of colon cancer actually seems astonishingly low.

Of course tissues at great risk also experience strong selection pressures to develop cancer suppressing strategies. Consider the repeating unit that forms the building block of the colon - the crypt. Within each crypt is a large population of differentiated cells with little or no proliferative capacity that are constantly renewed by pluripotent, tightly regulated stem cells confined to a small niche (Fig 1).

Of particular evolutionary interest is that normal functioning of the crypt requires cells to move from the site of proliferation to the site of differentiated function - a property shared by many epithelial tissues. We propose this organizational structure is a critical, evolved cancer prevention strategy because the movement from the stem cell niche to the basement membrane exposes the cells to two entirely different microenvironments and, hence, adaptive landscapes. The net effect is to impose a requirement for $t w o$ rather than one heritable change to even begin evolution toward a colon cancer. For example, a stem cell mutation that allows its differentiated daughter cells to crowd together on the basement membrane may produce a large, disordered population within the crypt. However, this mutation will not, by itself, produce a cancer because it does not alter proliferation of stem cells within their niche. That is, the mutation is not "rewarded" by increased proliferation of the stem cell line that initiated the population.

Similarly, a mutation that increases the proliferation of a stem cell will not form a cancer because the mutation confers increased fitness only in the distinct environment of the niche. If the mutant cells enter the basement membrane, they are subject to growth constraints to which they are not adapted. In the absence of proliferation in the crypt, this population cannot achieve a size necessary for further evolution.

Thus, we propose the "first step" of carcinogenesis is actually two steps - a stem-like cell capable of proliferation must move out of its niche onto the basement membrane and it must posses a mutation that allows continued proliferation despite membrane growth constraints. The latter is consistent with observations that APC mutations are among the first events in carcinogenesis, as this results in increased proliferation in the crypt. However, an equally important "first" event is a heritable change in the stem cell that allows its daughter cells to move onto the basement membrane while retaining an immature, proliferative phenotype (a kind of cellular neoteny). Note that similar results can be obtained by failure of the tissue control mechanism due to senescence or chronic inflammation that produce a loss of function mutations in the crypt myoepithelial cells, which operate as "gate-keepers".

In summary, multicellular organisms must evolve localized cancer prevention strategies and understanding these mechanisms is essential to defining the first step(s) of carcinogenesis. The organizational structure of normal, proliferating epithelium is one such strategy. The physical separation of stem cells and differentiated cells imposes a requirement for two independent heritable events thus reducing the probability of cancer initiation prior to the host's reproductive age.

\section{Acknowledgments}

This work was funded by:

Moffitt Cancer Center Physical Sciences-Oncology Center NIH/NCI 1U54CA143970-01 


\section{References}

1. Tanaka T. Colorectal carcinogenesis: Review of human and experimental animal studies. J Carcinog. 2009; 8:51-19.

2. Humphries A, Wright NA. Colonic crypt organization and tumorigenesis. Nat Rev Cancer. 2008; 8(6):415-24. [PubMed: 18480839]

3. Michod RE, Roze D. Cooperation and conflict in the evolution of multicellularity. Heredity. 2001; 86:1-7. [PubMed: 11298810] 

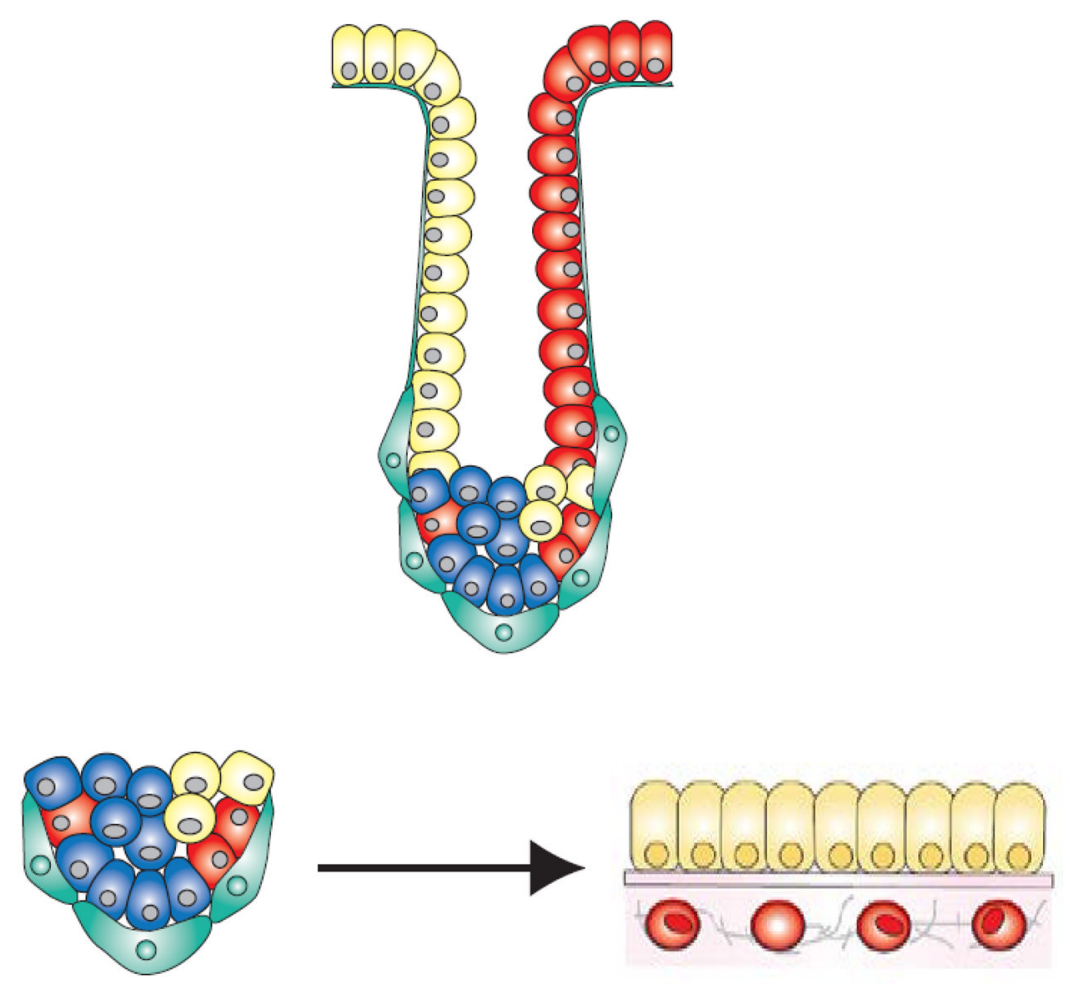

Figure 1.

An evolutionary model of a colon crypt highlighting the two distinctive adaptive landscapes: 1. The stem cell niche at the base of the crypt which is limited volume containing a small number of stem cells in close communication with distinctive myoepithelial cells. 2 . The much larger region in the remained of the crypt in which the daughter cells move along the basement membrane receiving signals from vascular factors and scattered mesenchymal cells. Normal crypt function requires cells to move from the stem cell niche adaptive landscape to that of the functioning region in the crypt. The initiation of carcinogenesis requires adaptations to growth constraints in each (very different) microenvironment. This tissue architecture in which the stem cells are isolated to an environment different from their differentiated daughters is probably a cancer prevention strategy evolved by multicellular organisms. 